\title{
Arthrogryposis of left upper limb hypoplasia and asymmetry
}

\section{Moawad S, Arevalo S, Zahouani T* and Rajegowda B \\ Department of Pediatrics, Lincoln Medical and Mental Health Center, USA}

We performed an assessment of a baby boy born early term, appropriate for gestational age at 38 weeks by caesarean section due to breech position. This was the $11^{\text {th }}$ pregnancy of the $30 \mathrm{y} / \mathrm{o}$ mother and all her other children had no problems during or after birth. There was no family history of congenital or limb malformation. The mother had history of Marijuana and alcohol use during that pregnancy and she had inadequate prenatal care with only three prenatal visits with one normal prenatal ultrasound in the second trimester. On physical examination, the baby had left upper limb hypoplasia with the left arm and forearm $1 \mathrm{~cm}$ shorter than the right arm and forearm with wasting of muscles and limitation of mobility. The left hand was hypoplastic with contractures, the five fingers were proportionally smaller, hypoplastic with syndactyly (Figure 1, 2 and 3). The rest of the physical examination was normal. The urine toxicology test of the baby and the mother was positive for Tetrahydrocannabinol (THC). Genetic testing with chromosomal analysis and microarray showed a normal chromosomal pattern without deletions or duplications. The patient was seen by rehabilitation services and will be followed by them along with and pediatric orthopedic for further intervention and management.

Arthrogryposis multiplex congenita distal type 1 (AMCD1), is characterized by one or more muscle and joint contractures, causing muscle shortening and inability to perform active extension and flexion in the affected joint or joints [1]. The exact incidence is unknown but reported to be 1-5/10000 births [1]. However, the involvement of upper extremity is rare compared to the lower extremity. The

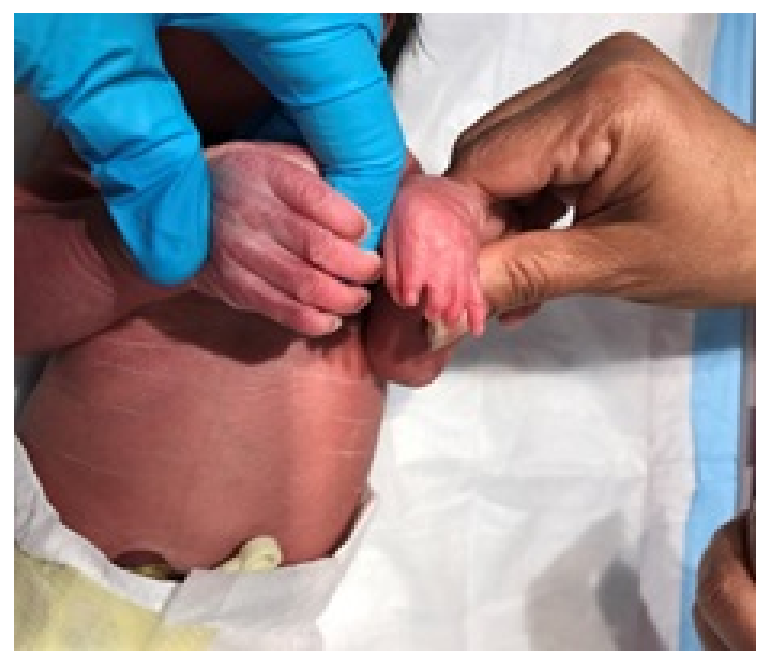

Figure 1. Left hand hypoplastic with 5 fingers proportionally smaller

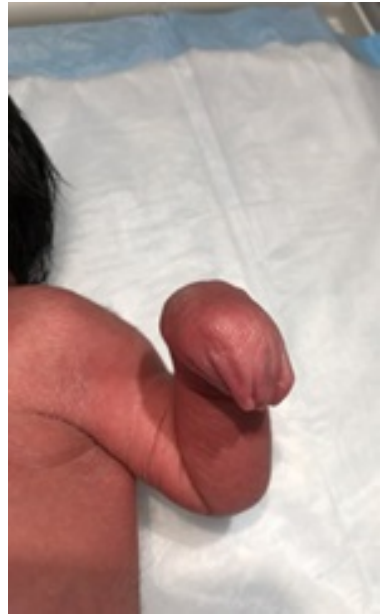

Figure 2. Left hand hypoplastic with contracture

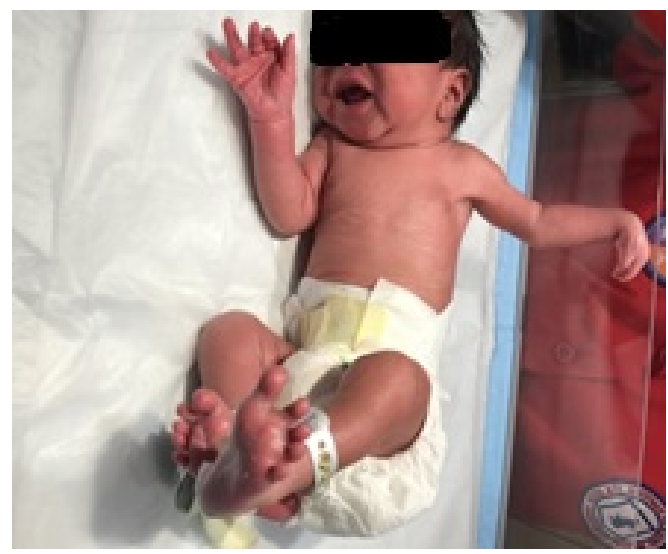

Figure 3. Left arm and forearm shorter than right arm and forearm

causes of this disorder can be related to environmental factors such as decreased intrauterine movement, oligohydramnios, defects in the fetal blood supply, hyperthermia, limb immobilization, viral infections and myasthenia gravis [1]. AMCD1 can also be caused by intrinsic factors such as molecular, muscle and connective tissue development disorders or neurological abnormalities [1]. Interestingly, the infant's

Correspondence to: Tarik Zahouani, Department of Pediatrics, Lincoln Medical Center, 237 East 149th St Bronx, NY 10451, USA, Tel: 718-579-5030; E-mail: Tarikzahouani@gmail.com

Received: July 03, 2017; Accepted: August 04, 2017; Published: August 07, 2017 
exposure to THC may cause decreased blood supply due to the increase in carboxyhemoglobin level, which impairs oxygen transfer in the lung, reduces the oxygen-carrying capacity of blood and releases oxygen from hemoglobin [2]. But the relationship of THC toxicity to this disorder was not proved. Also, the use of alcohol during pregnancy may produce fetal alcohol syndrome but our baby did not show any features suggestive of that syndrome. We believe some environmental factors played a role in this disorder which requires a complex treatment for improving the physical and functional capacity.

\section{References}

1. Bamshad M, Van Heest AE, Pleasure D. (2009) Arthrogryposis: A Review and Update. $J$ Bone Joint Surg Am 91: 40-46. [Crossref]

2. Forrester MB and Merz RD (2007) Risk of selected birth defects with prenatal illicit drug use, Hawaii, 1986-2002. J Toxicol Environ Health A. 70: 7-18. [Crossref]

Copyright: $\odot 2017$ Moawad S. This is an open-access article distributed under the terms of the Creative Commons Attribution License, which permits unrestricted use, distribution, and reproduction in any medium, provided the original author and source are credited. 